\title{
Evaluación mediante tres técnicas de susceptibilidad a fluconazol en especies de Candida aisladas en pacientes con infecciones invasoras. Bogotá - Colombia
}

\author{
Nidia A. Torres D., Carlos A. Álvarez M. y Martín A. Rondón S.
}

\section{Fluconazole susceptibility of invasive Candida sp isolates as determined by three methods. Bogota - Colombia}

The frequency of invasive mycosis caused by Candida sp has increased significantly in immunocompromised patients and in those under medical/surgical treatment; along with it, the use of azoles has fostered resistance. Objective: To compare in vitro susceptibility techniques, susceptibility to fluconazol was determined by the M27-A2 method of CLSI (Clinical Laboratory Standard Institute), the disk diffusion technique in 90 invasive Candida sp isolated species (49 C. albicans, 30 C. tropicalis, 5 C. parapsilosis, 4 C. guilliermondii and 2 C. lusitaniae) and the E-test in 16 isolated. Results: Over 90\% of strains were susceptible to fluconazol. A straight correlation between disk-diffusion technique and the E-test was observed regarding the reference method ( 0.944 and 0.938 , respectively). Conclusion: In our institution the use of fluconazol for invasive candidiasis is recommended, as well as the routine use of the disk-diffusion or E-test techniques in high complex hospitals in Colombia.

Key words: Candida, fluconazole, fungal drugs sensitivity tests, antifungal agents.

Palabras clave: Candida, fluconazol, pruebas de susceptibilidad antifúngica, antimicóticos.

\section{Introducción}

$\mathrm{E}$ n las últimas décadas, la frecuencia de micosis invasoras en pacientes inmunocomprometidos o con tratamientos médico-quirúrgicos ha aumentado significativamente. Otros factores que han incidido con el aumento de las infecciones fúngicas son el uso indiscriminado de antibacterianos de amplio espectro, catéteres vasculares y nutrición parenteral ${ }^{1}$. Este tipo de micosis son ocasionadas, en la mayoría de los casos, por levaduras del género Candida ${ }^{2}$.

De esta situación surgió la necesidad de utilizar antimicóticos como polienos, fluocitosina, imidazoles y triazoles (fluconazol e itraconazol), para profilaxis o terapia antifúngica o para la terapia empírica. Sin embargo, estas prácticas terapéuticas han generado cambios epidemiológicos entre los que se destacan la aparición de cepas que han desarrollado resistencia secundaria a los antifúngicos y la sustitución de algunas especies susceptibles por otras con resistencia intrínseca ${ }^{3,4}$.

En los últimos años se han estandarizado varias técnicas in vitro para la detección de la resistencia de los agentes etiológicos, que muestran correlación con la evolución clínica de los enfermos. Por eso, parece cada vez más importante conocer el perfil de susceptibilidad de las cepas clínicas y el espectro de acción de los antifúngicos ${ }^{4,5}$. En términos de costo real, las pruebas de susceptibilidad, exactas y precisas, son necesarias con el fin de establecer la posible eficacia clínica y orientar al médico en su decisión terapéutica ${ }^{2,6,7}$. Sin embargo, esta labor se había dificultado por la falta de una adecuada estandarización de las técnicas de estudio hasta la elaboración y publicación del documento M27-A2 por parte del Clinical Laboratory Standard Institute (CLSI) antes National Committee for Clinical Laboratory Standards (NCCLS). Debido a ciertas limitaciones de este método se han desarrollado otras alternativas como las técnicas basadas en caldo, los métodos colorimétricos comerciales Sensititre Yeast One $^{\circledR}$, Fungitest ${ }^{\circledR}$ y ASTY $^{\circledR}$ y técnicas basadas en la difusión en agar, como la epsilometría E-test ${ }^{\circledR}$ y el uso de discos que han sido ampliamente evaluados en levaduras con los antimicóticos actuales de uso común. La aceptación de uno u otro método ha estado condicionada por la ineludible relación costo/benefi$\mathrm{cio}^{2,3}$.
Hospital Universitario San Ignacio y Pontificia Universidad Javeriana, Bogotá, Colombia (NATD, CAAM).

Pontificia Universidad Javeriana, Bogotá, Colombia (MARS)

Recibido: 7 julio 2008 Aceptado: 3 diciembre 2008

Los autores declaran no tener conflictos de interés.

Correspondencia a: Nidia Alexandra Torres D. niditorres@yahoo.com 


\section{Objetivos}

- Comparar la determinación de susceptibilidad in vitro a fluconazol mediante las técnicas de microdilución en caldo M27-A2, difusión de discos en agar y E-test ${ }^{\circledR}$ en aislados de Candida recuperados de procesos infecciosos invasores en un hospital de tercer nivel, con el fin de elegir la técnica que fuera posible implementar de rutina en este tipo de instituciones.

- Conocer la situación real de la resistencia antifúngica en el hospital.

- Facilitar con los datos epidemiológicos, hasta el momento desconocidos para estos microorganismos, la implementación de programas de vigilancia en nuestro país.

\section{Material y Método}

Cepas en estudio. En el Hospital Universitario San Ignacio (HUSI) de 300 camas totales y 45 camas en Unidad de Cuidado Intensivo (UCI), a partir de los 142 aislados invasores recuperados durante el período comprendido entre el 20 de febrero de 2003 y 31 de diciembre de 2004, se recolectaron para el estudio por conveniencia 90 aislados de Candida sp, seleccionados usando una tabla con números aleatorios. Las muestras se agruparon de acuerdo con la fuente en: sangre, líquidos corporales (cefalorraquídeo, peritoneal, amniótico, pericárdico, sinovial, ascítico, abdominal, pancreático), y secreciones de absceso abdominal, tejidos blandos -como cuello, cadera, drenaje y secreción pseudocapsular-, pulmón, pleura, mediastino y páncreas.

De los aislados cultivados durante 48 horas en agar glucosado de Sabouraud (Referencia 01-165, Scharlau), se realizó una suspensión del microorganismo en $6 \mathrm{~mL}$ de agua destilada estéril y se colocó en dos frascos (3 $\mathrm{mL}$ cada uno) que fueron almacenados a temperatura ambiente. Para recuperar los aislados y obtener cultivos puros se inoculó el contenido de los frascos en placas de agar glucosado de Sabouraud y se incubó a $30 \pm 2{ }^{\circ} \mathrm{C}$ durante 24 horas. Para confirmar la identificación de las especies C. albicans y $C$. tropicalis se realizó una nueva resiembra de 24 horas a la misma temperatura en medio de CHROMagar Candida ${ }^{\mathbb{R}}$ (Becton Dickinson, Sparks, MD), a las 79 cepas previamente identificadas en el trabajo de rutina del HUSI, que incluye prueba de tubo germinal, prueba micromorfológica e identificación bioquímica con el equipo automatizado de MicroScan $^{\circledR}$ (Dade Behring, USA). Los aislados de C. parapsilosis, C. guilliermondii y C. Iusitaniae se inocularon nuevamente en paneles de identificación de MicroScan ${ }^{\circledR}$ para verificar la especie, dada la imposibilidad del medio CHROMagar para identificar estas especies ${ }^{8,9}$.

Las cepas para el control de calidad C. albicans (ATCC 66027) y C. tropicalis (ATCC 66029) fueron incluidas en cada montaje de los paneles de identificación.

Pruebas de susceptibilidad in vitro. Método de referencia de micro-dilución en caldo M27-A2. La prueba de susceptibilidad para fluconazol fue realizada siguiendo las indicaciones del documento de microdilución en caldo M27-A2 del CLSI ${ }^{10}$, excepto el método de lectura, para lo cual fue utilizada la espectrofotometría según European Committee on Antimicrobial Susceptibility Testing (EUCAST). La lectura espectrofotométrica se realizó a una longitud de onda de 530 nm y correspondió al 50\% de inhibición del crecimiento. Los puntos de corte utilizados para la interpretación fueron seguidos según el documento del CLSI ${ }^{10}$. Método de difusión de disco en agar. Se utilizó sensidiscos de $25 \mu \mathrm{g} / \mathrm{mL}$ de fluconazol, agar Mueller Hinton (BBL Cockeysville, MD) $+2 \%$ de glucosa $+0,5$ $\mu \mathrm{g} / \mathrm{ml}$ azul de metileno (medio GMB). El procedimiento y los puntos de corte utilizados siguieron las indicaciones del documento M44-P del CLSI ${ }^{11}$. Prueba de E-test. El número de aislados probados por esta técnica fue de 16 (seleccionados utilizando una tabla con números aleatorios). Se realizó el procedimiento y los puntos de corte utilizados fueron los indicados por el fabricante (AB Biodisk, Estocolmo, Suecia $)^{12}$. El medio empleado fue RPMI $-1640+2 \%$ glucosa + MOPS + $1,5 \%$ Bacto agar (SIGMA).

Estandarización de las pruebas empleando las cepas control ATCC. Los resultados de las CIMs, obtenidas en la técnica de microdilución, difusión de discos en agar y E-test ${ }^{\circledR}$ para las cepas control ATCC C. krusei 6258 y C. parapsilosis 22019 a las 24 y 48 horas se encontraron dentro de los rangos definidos por el CLSI con el antifúngico evaluado (Documento M 27-A2, documento M44-P, Biodisk).

Análisis estadístico. La comparación de las técnicas de micro-dilución M27-A2, difusión de disco en agar y E-test ${ }^{\circledR}$ se hizo mediante la evaluación del porcentaje de acuerdo y del grado de concordancia entre ellas mediante el índice de kappa. Para comparar las frecuencias de susceptibilidad de $C$. albicans y $C$. tropicalis a fluconazol se utilizó la prueba de chi cuadrado de Pearson. Se consideró una diferencia estadísticamente significativa cuando $\mathrm{p}<0,05$. Los cálculos se realizaron con el paquete estadístico STATA $7^{\circledR}$.

Características clínicas de los pacientes. Se realizó una revisión de las historias clínicas teniendo en cuenta los factores predisponentes, el tratamiento dado dos semanas antes de la fecha de la recuperación de la levadura en el laboratorio. En los casos donde se 
administró antifúngico se investigó el tipo de antifúngico dado. Además se determinó el porcentaje de mortalidad.

\section{Resultados}

Aislados. En el HUSI de las 90 cepas de Candida provenientes de muestras invasoras $57,8 \%$ fueron obtenidas de sangre, $25,5 \%$ provenían de líquidos corporales y $16,7 \%$ de secreciones. La distribución de las especies de Candida aisladas se muestran en la tabla 1 .
Tabla 1. Distribución de especies de Candida sp aisladas de muestras de procesos invasores

\begin{tabular}{|lcc|}
\hline Especies & n & $\%$ \\
\hline Candida albicans & 49 & 54,5 \\
\hline Candida tropicalis & 30 & 33,3 \\
\hline Candida parapsilosis & 5 & 5,6 \\
\hline Candida guilliermondii & 4 & 4,4 \\
\hline Candida lusitaniae & 2 & 2,2 \\
Total & 90 & 100 \\
\hline
\end{tabular}

\begin{tabular}{|c|c|c|c|c|c|c|c|c|}
\hline *ID & $\underset{(\mu \mathrm{g} / \mathrm{ml})}{\mathrm{CIM}}$ & **Interpretación & *ID & $\underset{(\mu \mathrm{g} / \mathrm{ml})}{\mathrm{CIM}}$ & **Interpretación & *ID & $\underset{(\mu \mathrm{g} / \mathrm{ml})}{\mathrm{CIM}}$ & **Interpretación \\
\hline 468780 & $<0,25$ & 1 & 547615 & $<0,25$ & 1 & 590087 & $<0,25$ & 1 \\
\hline 471165 & $<0,25$ & 1 & 551834 & $<0,25$ & 1 & 591381 & $<0,25$ & 1 \\
\hline 477084 & $<0,25$ & 1 & 562123 & $<0,25$ & 1 & 597012 & $<0,25$ & 1 \\
\hline $476333 \mathrm{~A}$ & $<0,25$ & 1 & 542172 & 32 & 3 & 610469 & $<0,25$ & 1 \\
\hline 482764 & $<0,25$ & 1 & 544602 & $<0,25$ & 1 & 603743 & $<0,25$ & 1 \\
\hline 560255 & $<0,25$ & 1 & 546472 & $<0,25$ & 1 & 480616 & $<0,25$ & 1 \\
\hline 481636 & $<0,25$ & 1 & 546215 & $<0,25$ & 1 & 606141 & $<0,25$ & 1 \\
\hline 498252 & 16 & 3 & 546936 & $<0,25$ & 1 & 614367 & $<0,25$ & 1 \\
\hline 478204 & $<0,25$ & 1 & 547719 & $<0,25$ & 1 & 612162 & $<0,25$ & 1 \\
\hline 528253 & $<0,25$ & 1 & 545101 & $<0,25$ & 1 & 614938 & $<0,25$ & 1 \\
\hline 550665 & $<0,25$ & 1 & 554194 & $<0,25$ & 1 & 617153 & $<0,25$ & 1 \\
\hline 501136 & $<0,25$ & 1 & 554667 & $<0,25$ & 1 & 619323 & $<0,25$ & 1 \\
\hline 500553 & $<0,25$ & 1 & 554700 & $<0,25$ & 1 & 622676 & $<0,25$ & 1 \\
\hline 512096 & $<0,25$ & 1 & 563579 & $<0,25$ & 1 & 634413 & $<0,25$ & 1 \\
\hline 501416 & $<0,25$ & 1 & 558915 & $<0,25$ & 1 & 637630 & $<0,25$ & 1 \\
\hline 501301 & $<0,25$ & 1 & 568236 & $<0,25$ & 1 & 634932 & $<0,25$ & 1 \\
\hline 518163 & $<0,25$ & 1 & 562108 & 8 & 1 & 638165 & $<0,25$ & 1 \\
\hline 509912 & $<0,25$ & 1 & 552665 & 1 & 1 & 639209 & $<0,25$ & 1 \\
\hline 511529 & $<0,25$ & 1 & 570300 & $<0,25$ & 1 & 639489 & $<0,25$ & 1 \\
\hline 513136 & $<0,25$ & 1 & 579508 & $<0,25$ & 1 & 645169 & 4 & 1 \\
\hline 513718 & 0,25 & 1 & 579779 & $<0,25$ & 1 & 653056 & $<0,25$ & 1 \\
\hline 522077 & $<0,25$ & 1 & 576318 & $<0,25$ & 1 & 657998 & $<0,25$ & 1 \\
\hline 517821 & $<0,25$ & 1 & 578240 & $<0,25$ & 1 & 669139 & $<0,25$ & 1 \\
\hline 528318 & $<0,25$ & 1 & 592057 & $<0,25$ & 1 & 671331 & $<0,25$ & 1 \\
\hline 528909 & $<0,25$ & 1 & 582102 & 2 & 1 & 9003035 & $<0,25$ & 1 \\
\hline 520532 & $<0,25$ & 1 & 592247 & $<0,25$ & 1 & 9004321 & $<0,25$ & 1 \\
\hline 815004 & $<0,25$ & 1 & 585109 & $<0,25$ & 1 & $9006355 \mathrm{~A}$ & $<0,25$ & 1 \\
\hline 527683 & $<0,25$ & 1 & 586393 & $<0,25$ & 1 & 9015582 & $<0,25$ & 1 \\
\hline 561803 & $<0,25$ & 1 & 587237 & $<0,25$ & 1 & 9019023 & $<0,25$ & 1 \\
\hline $541214 \mathrm{~A}$ & $<0,25$ & 1 & 587500 & $<0,25$ & 1 & 9021680 & $<0,25$ & 1 \\
\hline
\end{tabular}




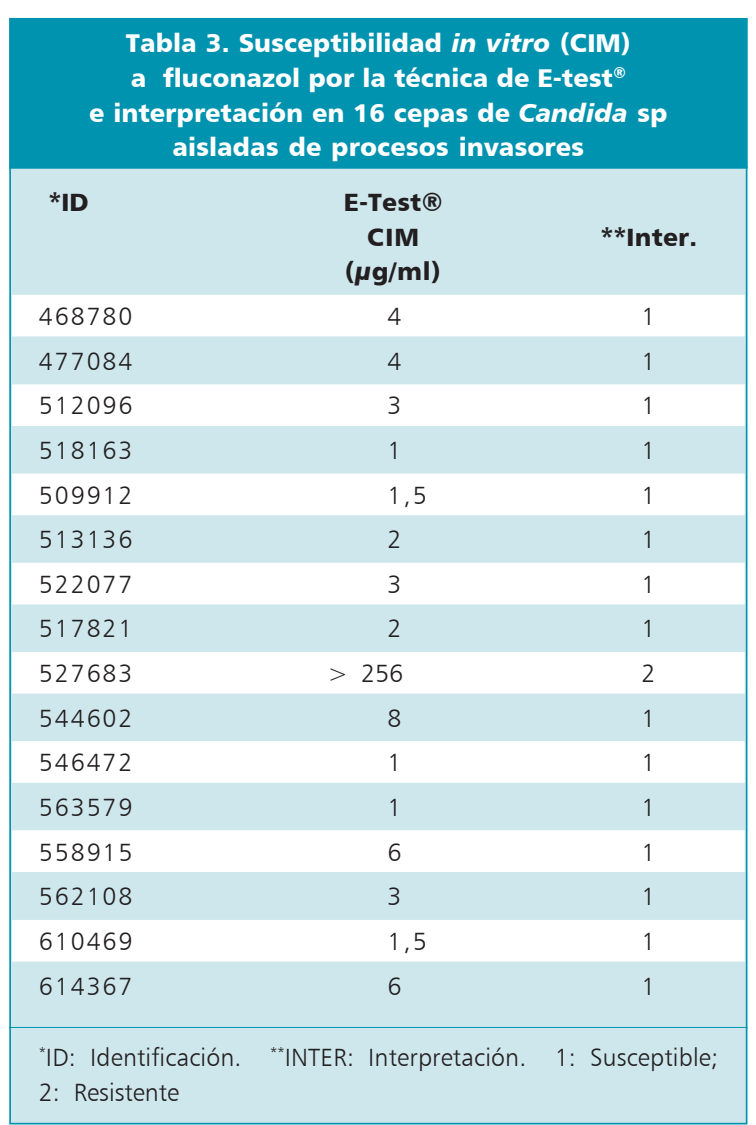

La confirmación de especie mediante CHROMagar Candida de 79 cepas demostró una adecuada identificación de las especies C. albicans (verde) y C. tropicalis (azul) sin encontrar resultados dudosos que sugirieran la realización de pruebas adicionales. Adicionalmente, para las 11 cepas restantes de C. parapsilosis, C. lusitaniae, C. guilliermondii, especies que no diferencia este medio, el panel de MicroScan ${ }^{\circledR}$ las verificó respecto a los resultados obtenidos previamente dentro de la rutina del HUSI.

Determinación de la susceptibilidad in vitro y concordancia entre métodos (Tablas 2 a 6).

Inicialmente se hizo el cálculo del porcentaje de acuerdo entre las técnicas evaluadas observándose 94,4 y $93,8 \%$ de acuerdo entre la difusión en agar y E test ${ }^{\circledR}$ respectivamente, con el método de referencia M27-A2. Sin embargo, encontramos que no existía un buen grado de concordancia entre las técnicas, pues los valores de los índices de kappa fueron muy bajos $(\mathrm{k}<0,001)$.

Por otra parte, el análisis estadístico chi-cuadrado de Pearson no encontró diferencias estadísticamente significativas $(\mathrm{p}>0,05)$ entre la susceptibilidad de $C$. albicans y C. tropicalis, las dos especies más frecuentemente aisladas, a fluconazol.

El $82,9 \%$ de los pacientes había recibido antibacterianos previamente. El $64,4 \%$ (n: 58 ) recibió tratamiento utilizando fluconazol en $83,0 \%$ de ellos (n: 48) y anfotericina B 17\% (n: 10). Se observó una mortalidad del $38 \%$ (Tabla 7).

\section{Discusión}

Las especies de Candida se encuentran entre los agentes etiológicos más comunes de infección nosocomial invasora y están asociadas con una alta mortalidad ${ }^{13}$ especialmente en los pacientes de las Unidades de Cuidado Intensivo (UCI) de las instituciones de salud $^{14-17}$.

En nuestro hospital, la tasa de infección sanguínea por Candida sp es de 2,9\%; sin embargo, la mortalidad asociada a este tipo de infecciones, tanto sanguíneas como de otro origen invasor, sigue siendo alta (38\%), similar a lo descrito en la literatura científica, a pesar del manejo antimicótico adecuado. Por otra parte, los factores predisponentes encontrados en este estudio para la presencia de una candidiasis invasora reflejan lo descrito en otros estudios (antibacterianos previos, nutrición parenteral, cirugía abdominal, etc) y, aunque este estudio no fue diseñado para evaluar el impacto del riesgo con dichos factores, su alta frecuencia permite colegir la importancia de los mismos.

El orden de frecuencias de las especies de Candida en este estudio se correlaciona con lo encontrado por el Grupo para el Control de la Resistencia Antimicrobiana en Bogotá (GREBO). Ellos comunicaron 1.194 aislados micóticos entre 2001 y 2002 provenientes de ocho hospitales, demostrando que los hongos más frecuentemente aislados en UCI fueron: $C$. albicans (57\%), C. tropicalis (14\%), C. guilliermondii (5\%), Trichosporon sp. (3\%) y hubo Candida sp en 7\%. En las otras unidades clínicas los más frecuentes fueron C. albicans (54\%), C. tropicalis (15\%), C. neoformans (4\%), Trichosporon sp. (21\%) y Candida sp. $(3 \%)^{18}$. En ambos estudios se demuestra que $C$. tropicalis se encuentra con mayor frecuencia que otras especies de Candida no albicans, similar a lo descrito en Israel (C. tropicalis $25 \%)^{19}$. Este hallazgo es diferente a lo descrito en el programa de vigilancia SENTRY que además de América Latina incluye Norteamérica y otro realizado en Italia, en los cuales C. albicans sigue siendo la especie predominante, pero en segundo lugar se ubica C. glabrata. Candida tropicalis se encuentra en una frecuencia menor a $10 \%$, ocupando un quinto lugar ${ }^{20-23}$.

En el estudio de vigilancia de resistencia ARTEMIS 1997-2005, que incluye aislados provenientes de la 


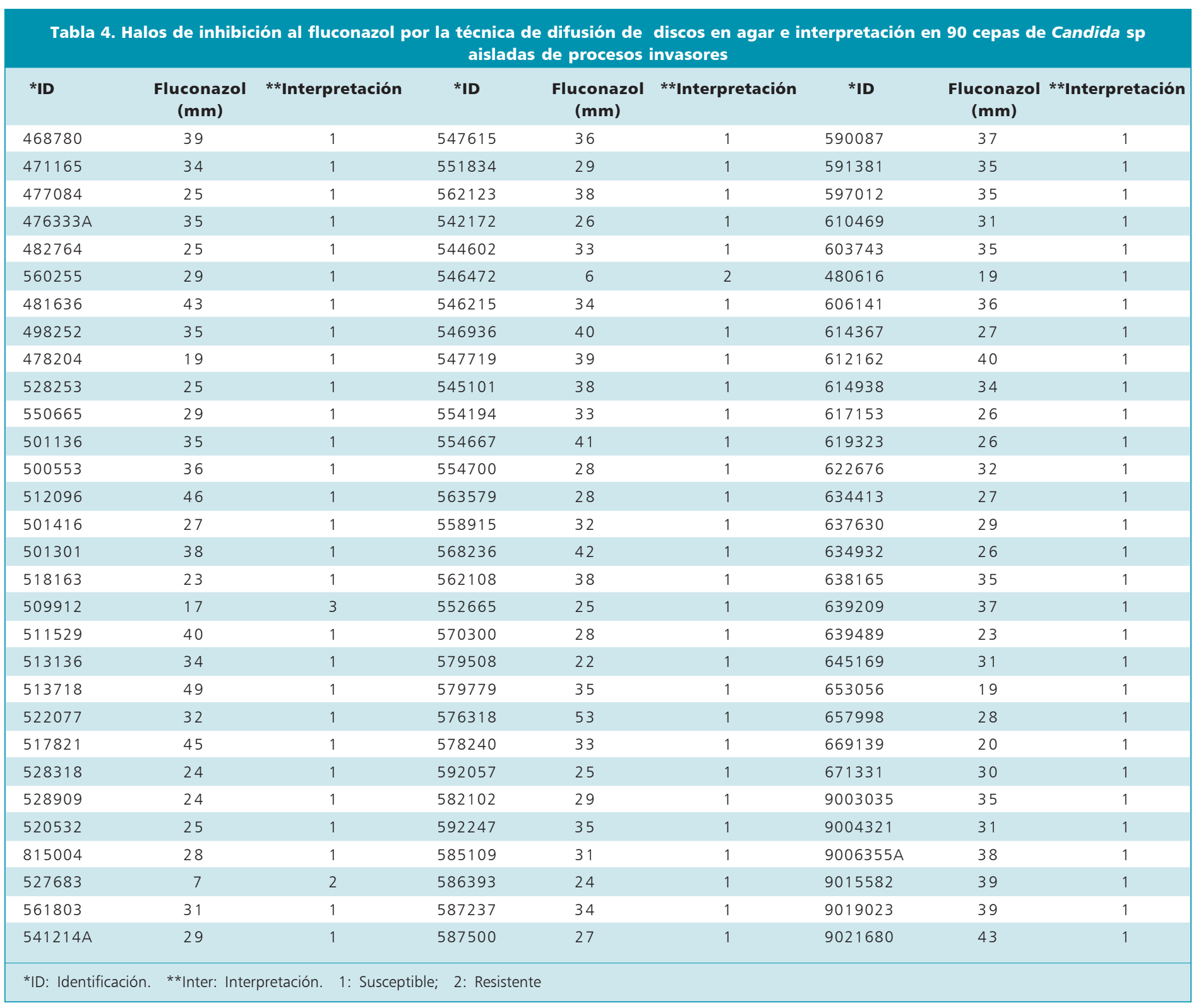

región Pacífico-Asia, Latinoamérica, Europa, Oriente Medio/África y Norteamérica, se observó una susceptibilidad al fluconazol superior a $90 \%$ en los aislados de Candida evaluados ${ }^{24}$. En un estudio colombiano, en pacientes con cáncer, $82 \%$ de los aislados clínicos de levaduras eran susceptibles a fluconazol ${ }^{25}$ similar a lo descrito en otra publicación de resistencia antimicótica en la región andina (CELA, Colombia, Ecuador y Venezuela) en la cual $88,1 \%$ de los aislados presentaron susceptibilidad a fluconazol ${ }^{26} \mathrm{y}$ otra llevada a cabo en una UCI de un hospital del oriente de Colombia en el que no se detectó resistencia in vitro a fluconazol ${ }^{27}$. Otro estudio colombiano realizado con cepas de
Candida sp obtenidas de la mucosa oral en pacientes con SIDA mostró que $72,9 \%$ de los aislados eran susceptibles a fluconazol, 6,3\% tenían SDD (susceptibilidad dosis-dependiente) y $20,8 \%$ eran resistentes ${ }^{28}$. Estos estudios en general sugieren que la respuesta a

\begin{tabular}{|c|c|c|c|c|c|}
\hline Antimicótico & $\begin{array}{l}\text { Rango } \\
(\mu \mathrm{g} / \mathrm{ml})\end{array}$ & $\begin{array}{c}\mathrm{CIM}_{90} \\
(\mu \mathrm{g} / \mathrm{ml})\end{array}$ & $\% \mathbf{S}$ & $\%$ SDD & $\% \mathbf{R}$ \\
\hline Fluconazol & $<0,25-32$ & $<0,25$ & $97,8 \%$ & $2,2 \%$ & $0 \%$ \\
\hline
\end{tabular}


Tabla 6. Comparación de las técnicas M27-A2 y difusión de discos en agar en cepas de Candida sp. aisladas de procesos invasores

\begin{tabular}{|c|c|c|c|c|c|c|c|c|c|}
\hline Especie & $\begin{array}{l}\text { Técnica } \\
\text { CIM } \\
(\mu \mathrm{g} / \mathrm{ml})\end{array}$ & $\begin{array}{c}\text { M27-A2 } \\
\text { Inter- } \\
\text { pretación }\end{array}$ & $\begin{array}{c}\text { Difusiór } \\
\text { Halo } \\
\text { (mm) }\end{array}$ & $\begin{array}{c}\text { en agar } \\
\text { Inter- } \\
\text { pretación }\end{array}$ & Especie & $\begin{array}{l}\text { Técnica } \\
\text { CIM } \\
(\mu \mathrm{g} / \mathrm{ml})\end{array}$ & $\begin{array}{c}\text { M27-A2 } \\
\text { Inter- } \\
\text { pretación }\end{array}$ & $\begin{array}{c}\text { Difusión } \\
\text { Halo } \\
\text { (mm) }\end{array}$ & $\begin{array}{c}\text { en agar } \\
\text { Inter- } \\
\text { pretación }\end{array}$ \\
\hline C. albicans & $<0,25$ & 1 & 39 & 1 & C. albicans & 8 & 1 & 38 & 1 \\
\hline C. albicans & $<0,25$ & 1 & 34 & 1 & C. albicans & 1 & 1 & 25 & 1 \\
\hline C. albicans & $<0,25$ & 1 & 25 & 1 & C. albicans & $<0,25$ & 1 & 28 & 1 \\
\hline C. albicans & $<0,25$ & 1 & 35 & 1 & C. albicans & $<0,25$ & 1 & 22 & 1 \\
\hline C. tropicalis & $<0,25$ & 1 & 25 & 1 & C. albicans & $<0,25$ & 1 & 35 & 1 \\
\hline C. albicans & $<0,25$ & 1 & 29 & 1 & C. albicans & $<0,25$ & 1 & 53 & 1 \\
\hline C. albicans & $<0,25$ & 1 & 43 & 1 & C. parapsilosis & $<0,25$ & 1 & 33 & 1 \\
\hline C. albicans & 16 & 3 & 35 & 1 & C. albicans & $<0,25$ & 1 & 25 & 1 \\
\hline C. tropicalis & $<0,25$ & 1 & 19 & 1 & C. albicans & 2 & 1 & 29 & 1 \\
\hline C. guilliermondii & $<0,25$ & 1 & 25 & 1 & C. albicans & $<0,25$ & 1 & 35 & 1 \\
\hline C. albicans & $<0,25$ & 1 & 29 & 1 & C. tropicalis & $<0,25$ & 1 & 31 & 1 \\
\hline C. albicans & $<0,25$ & 1 & 35 & 1 & C. tropicalis & $<0,25$ & 1 & 24 & 1 \\
\hline C. tropicalis & $<0,25$ & 1 & 36 & 1 & C. albicans & $<0,25$ & 1 & 34 & 1 \\
\hline C. albicans & $<0,25$ & 1 & 46 & 1 & C. tropicalis & $<0,25$ & 1 & 27 & 1 \\
\hline C. guilliermondii & $<0,25$ & 1 & 27 & 1 & C. Iusitaniae & $<0,25$ & 1 & 37 & 1 \\
\hline C. tropicalis & $<0,25$ & 1 & 38 & 1 & C. albicans & $<0,25$ & 1 & 35 & 1 \\
\hline C. guilliermondii & $<0,25$ & 1 & 23 & 1 & C. albicans & $<0,25$ & 1 & 35 & 1 \\
\hline C. tropicalis & $<0,25$ & 1 & 17 & 3 & C. albicans & $<0,25$ & 1 & 31 & 1 \\
\hline C. tropicalis & $<0,25$ & 1 & 40 & 1 & C. tropicalis & $<0,25$ & 1 & 35 & 1 \\
\hline C. albicans & $<0,25$ & 1 & 34 & 1 & C. tropicalis & $<0,25$ & 1 & 19 & 1 \\
\hline C. tropicalis & 0,25 & 1 & 49 & 1 & C. tropicalis & $<0,25$ & 1 & 36 & 1 \\
\hline C. albicans & $<0,25$ & 1 & 32 & 1 & C. albicans & $<0,25$ & 1 & 27 & 1 \\
\hline C. albicans & $<0,25$ & 1 & 45 & 1 & C. tropicalis & $<0,25$ & 1 & 40 & 1 \\
\hline C. tropicalis & $<0,25$ & 1 & 24 & 1 & C. albicans & $<0,25$ & 1 & 34 & 1 \\
\hline C. albicans & $<0,25$ & 1 & 24 & 1 & C. tropicalis & $<0,25$ & 1 & 26 & 1 \\
\hline C. tropicalis & $<0,25$ & 1 & 25 & 1 & C. albicans & $<0,25$ & 1 & 26 & 1 \\
\hline C. tropicalis & $<0,25$ & 1 & 28 & 1 & C. albicans & $<0,25$ & 1 & 32 & 1 \\
\hline C. tropicalis & $<0,25$ & 1 & 7 & 2 & C. parapsilosis & $<0,25$ & 1 & 27 & 1 \\
\hline C. albicans & $<0,25$ & 1 & 31 & 1 & C. parapsilosis & $<0,25$ & 1 & 29 & 1 \\
\hline C. albicans & $<0,25$ & 1 & 29 & 1 & C. albicans & $<0,25$ & 1 & 26 & 1 \\
\hline C. tropicalis & $<0,25$ & 1 & 36 & 1 & C. parapsilosis & $<0,25$ & 1 & 35 & 1 \\
\hline C. tropicalis & $<0,25$ & 1 & 29 & 1 & C. tropicalis & $<0,25$ & 1 & 37 & 1 \\
\hline C. albicans & $<0,25$ & 1 & 38 & 1 & C. tropicalis & $<0,25$ & 1 & 23 & 1 \\
\hline C. lusitaniae & 32 & 3 & 26 & 1 & C. albicans & 4 & 1 & 31 & 1 \\
\hline C. albicans & $<0,25$ & 1 & 33 & 1 & C. guilliermondii & $<0,25$ & 1 & 19 & 1 \\
\hline C. albicans & $<0,25$ & 1 & 6 & 2 & C. albicans & $<0,25$ & 1 & 28 & 1 \\
\hline C. tropicalis & $<0,25$ & 1 & 34 & 1 & C. tropicalis & $<0,25$ & 1 & 20 & 1 \\
\hline C. albicans & $<0,25$ & 1 & 40 & 1 & C. albicans & $<0,25$ & 1 & 30 & 1 \\
\hline C. albicans & $<0,25$ & 1 & 39 & 1 & C. albicans & $<0,25$ & 1 & 35 & 1 \\
\hline C. tropicalis & $<0,25$ & 1 & 38 & 1 & C. tropicalis & $<0,25$ & 1 & 31 & 1 \\
\hline C. albicans & $<0,25$ & 1 & 33 & 1 & C. parapsilosis & $<0,25$ & 1 & 38 & 1 \\
\hline C. albicans & $<0,25$ & 1 & 41 & 1 & C. tropicalis & $<0,25$ & 1 & 39 & 1 \\
\hline C. tropicalis & $<0,25$ & 1 & 28 & 1 & C. albicans & $<0,25$ & 1 & 39 & 1 \\
\hline C. albicans & $<0,25$ & 1 & 28 & 1 & C. albicans & $<0,25$ & 1 & 43 & 1 \\
\hline C. tropicalis & $<0,25$ & 1 & 32 & 1 & & & & & \\
\hline C. albicans & $<0,25$ & 1 & 42 & 1 & 1: Susceptible; & stente; $3: \mathrm{S}$ & & & \\
\hline
\end{tabular}


fluconazol de los aislados de Candida en la región andina se encuentra por encima de $80 \%$. En este trabajo, la susceptibilidad a fluconazol es muy alta, situación que podría ser explicada por el bajo consumo de este antimicótico; en el HUSI la política de uso prudente de antimicrobianos limita el uso de estos medicamentos en forma profiláctica, aun en pacientes con factores predisponentes; la recomendación para su uso se limita a tratamiento de forma empírica o dirigida.

Las especies que han demostrado resistencia a fluconazol, C. krusei y C. glabrata, no fueron aisladas en el presente estudio, demostrando su muy baja incidencia en la institución lo que puede ser explicado por un adecuado control en la administración de los antimicóticos que disminuye la selección de cepas, factor primordial en la selección de cepas resistentes ${ }^{19,29,30}$.

En la evaluación de fluconazol empleando los tres métodos se encontraron resultados similares de susceptibilidad y resistencia. Estudios previos demostraron que $96 \%$ de los resultados de E-test ${ }^{\circledR}$ y $97 \%$ de los resultados de la prueba de difusión se correlacionaban con los obtenidos por el método de referencia M27$\mathrm{A} 2^{6,31}$. Los resultados obtenidos indican que la técnica de difusión para fluconazol sirve para detectar los aislados susceptibles garantizando que el resultado es confiable, pero no para los resistentes ni SDD ya que el índice kappa da un resultado negativo, lo que sugiere la necesidad de confirmar por el método de referencia u otro método la CIM. Esta necesidad también fue encontrada en trabajos previos ${ }^{32-34}$.

Algunos estudios ${ }^{32-34}$ encontraron una correlación de $90 \%$ de la técnica de difusión con el método de referencia M27-A2. Cuando evaluaron la correlación para las categorías, encontraron $99,6 \%$ para susceptibilidad, $19,0 \%$ para SDD y $52,1 \%$ para resistencia lo que sugiere que los resultados de ambos métodos tienen una elevada correlación para especies de Candida susceptibles al fluconazol pero disminuye para las SDD y resistentes. La correlación de la técnica de E-test ${ }^{\circledR}$ con el método de referencia puede variar dependiendo del agente antifúngico probado, elección del medio de cultivo y la especie de levadura ensayada; se ha demostrado una disminución de la correlación con aislados de C. glabrata y $C$. tropicalis $^{35}$. En el presente estudio no fue posible concluir la utilidad de este método al evaluar las cepas resistentes, dado el bajo número de aislados ensayado con esta técnica y el número limitado de cepas resistentes.

Aunque los valores de concordancia son altos, los índices kappa observados indican que las técnicas evaluadas no reemplazan el método de referencia, pero es importante aclarar que esta afirmación está influenciada por el número disminuido de aislados resis-
Tabla 7. Características clínicas y factores de riesgo predisponentes de los pacientes con procesos invasores causados por Candida sp
Factor de riesgo predisponente

Cirugía general (abdomen)

Trasplante de órgano sólido (hígado)

Infección por VIH

Diabetes mellitus

Nutrición parenteral

Ventilación mecánica

Antimicrobianos previos

Enfermedades infecciosas concomitantes

Tratamiento antimicótico usado

Fluconazol

Anfotericina B

Mortalidad asociada a la candidiasis
Frecuencia (\%)

31,7

1,2

6,1

7,3

32,9

50,0

82,9

81,7

83

17

38 tentes para fluconazol y la cantidad limitada de resultados obtenidos por la técnica de E-test ${ }^{\circledR}$.

El análisis estadístico chi-cuadrado de Pearson no encontró diferencias significativas entre la susceptibilidad de C. albicans y C. tropicalis a fluconazol. Por las técnicas M27-A2 y difusión de discos en agar se demostró que las dos especies tienen un comportamiento similar de alta susceptibilidad a fluconazol. Estos resultados han sido encontrados en diferentes estudios como el realizado en la UCI de un hospital del oriente de Colombia con aislados de $C$. albicans y $C$. tropicalis ( $45 \%$ en ambos casos) con susceptibilidad in vitro de $100 \%$ a fluconazol, independientemente de la forma de infección y de otras variables clínicas ${ }^{27}$. Además, en otros estudios se encontró susceptibilidad de 92,1 y 90,0\% respectivamente ${ }^{26}$ y en el Programa de Vigilancia SENTRY encontraron 98,9\% de susceptibilidad para C. albicans y $100 \%$ para C. tropicalis con aislados de Canadá y Latinoamérica, que mostraron ser más susceptibles, tanto a fluconazol como a itraconazol, que los hallados en E.U.A ${ }^{23}$. Dichos resultados demuestran que la actividad de fluconazol permanece alta contra aislados de especies de C. albicans y C. tropicalis $^{36}$.

En conclusión, en nuestra institución recomendamos el uso de fluconazol para el tratamiento de infecciones micóticas invasoras y así mismo recomendamos la implementación de las técnicas de difusión con disco o E-test ${ }^{\circledR}$ para determinar la susceptibilidad de forma rutinaria en hospitales de alta complejidad de Colombia.

Agradecimientos. A Leonard Aguilar, profesor de Medicina Interna de la Pontificia Universidad Javeriana por su apoyo en la recolección de la información clínica. 


\section{Resumen}

La frecuencia de micosis invasoras causadas por Candida sp en pacientes inmunocomprometidos o con tratamientos médico-quirúrgicos ha aumentado y con ello, el uso de azoles, facilitando la aparición de resistencia. Objetivo: Comparar tres métodos de detección de susceptibilidad in vitro a fluconazol: el método de referencia M27-A2 del CLSI (Clinical Laboratory Standard Institute), técnica de difusión en disco a 90 aislados obtenidos en procesos infecciosos invasores (49 C. albicans; 30 C. tropicalis; 5
C. parapsilosis; 4 C. guilliermondii y 2 C. lusitaniae) y la epsilometría (E-test $\left.{ }^{\circledR}\right)$ en 16 aislados. Resultados: La susceptibilidad fue mayor a $90 \%$. Se encontró un buen porcentaje de concordancia entre las técnicas de difusión de disco y E-test ${ }^{\circledR}$ con respecto al método de referencia $(0,944$ y 0,938 , respectivamente). Conclusión: En nuestra institución se considera de elección fluconazol para tratar infecciones micóticas invasoras y recomendamos las técnica de difusión en disco o E-test ${ }^{\circledR}$ para determinar la susceptibilidad en la rutina en hospitales de alta complejidad en Colombia.

\section{Referencias}

1.- Neely M, Ghannoum M. The exciting future of antifungal therapy. Eur J Clin Microbiol Infect Dis 2000; 19: 897-914.

2.- Carrillo A, Abarca L, Quindós G. Métodos colorimétricos para la determinación de la sensibilidad in vitro a los antifúngicos. Rev Iberoam Micol 2001; 18: 150-5.

3.- Cuenca-Estrella M, Rodríguez-Tudela J. ¿Pueden basarse las indicaciones de los antifúngicos en los estudios de sensibilidad? Rev Iberoam Micol 2002; 19: 133-8.

4.- Johnson Elizabeth M. Issues in antifungal susceptibility testing. J Antimicrob Chemother 2008; 61 (Suppl). 1, il3-il8.

5.- Espinel-Ingroff A. Antifungal susceptibility testing. Clin Microbiol Newsletter 1996; 18: 161-7.

6.- Barry AL, Pfaller MA, Rennie RP, Fuchs PC, Brown SD. Precision and accuracy of fluconazole susceptibility testing by broth microdilution, Etest, and disk diffusion methods. Antimicrob Agents Chemother 2002; 46: 1781-4.

7.- Forrest G. Role of antifungal susceptibility testing in patient management. Curr Opin Infect Dis 2006; 6: 538-43.

8.- Klevay M, Ebinger A, Diekema D, Messer S, Hollis R, Pfaller M. Disk diffusion testing using Candida sp. colonies taken directly from CHROMagar medium may decrease time required to obtain results. J Clin Microbiol 2005; 43: 3497-9.

9.- Tan G, Peterson E. CHROMagar Candida medium for direct susceptibility testing of yeast from blood cultures. J Clin Microbiol 2005; 43: 1727-31.

10.- NCCLS (National Committee for Clinical Laboratory Standards). 2002. Reference method for broth dilution antifungal susceptibility testing of yeasts. Approved Standard-Second Edition. NCCLS document M27-A2 (ISBN 1-56238-469-4). NCCLS, 940 West Valley Road, Suite 1400, Wayne,
Pennsylvania 19087-1898 USA.

11.- NCCLS. 2003. Method for antifungal disks diffusion susceptibility testing of yeasts; proposed guideline. NCCLS Document M44-P (ISBN 1-56238-488-0). NCCLS, 940 West Valley Road, Suite 1400, Wayne, Pennsylvania 19087-1898 USA.

12.- Chryssantou E. Trends in antifungal susceptibility among Swedish Candida species bloodstream isolates from 1994 to 1998: comparison of E-test and the Sensititre YeastOne colorimetric antifungal panel with the NCCLS M27-A reference method. J Clin Microbiol 2001; 39: 4181-3.

13.- Gudlaugsson O, Gillespie S, Lee K, Vande-Berg J, Hu J, Messer S, et al. Attributable mortality of nosocomial candidemia, revisited. Clin Infect Dis 2003; 37: $1172-7$

14.- Berrouane Y, Herwaldt L, Pfaller M. Trends in antifungal use and epidemiology of nosocomial yeast infections in a university hospital. J Clin Microbiol 1999; 37: 531-7.

15.- Bustamante C. Treatment of Candida infection: a view from the trenches! Curr Opin Infect Dis 2005; 18: 490-5.

16.- Sabatelli F, Patel R, Mann P, Mendrick C, Norris C, Hare R, et al. In vitro activities of posaconazole, fluconazole, itraconazole, voriconazole, and amphotericin B against a large collection of clinically important molds and yeasts. Antimicrob Agents Chemother 2006; 50: 2009-15.

17.- Wenzel RP. Nosocomial candidemia: risk factors and attributable mortality. Clin Infect Dis 1995; 20: 1531-4.

18.- Cortés J, Leal A, Alvarez C. Frecuencia de aislamientos micóticos en hospitales de tercero y cuarto nivel en Bogotá, 20012002. Infectio 2003; 7: 110.

19.- Samra Z, Yardeni M, Peled N, Bishara J. Species distribution and antifungal susceptibility of Candida bloodstream isolates in a tertiary medical center in Israel. Eur J Clin Microbiol Infect Dis 2005; 24 :
592-5.

20.- St-Germain G, Laverdière M, Pelletier A, Bourgault M, Libman M, Lemieux C, et al. Prevalence and antifungal susceptibility of 442 Candida isolates from blood and other normally sterile sites: results of a 2-year (1996 to 1998) multicenter surveillance study in Quebec, Canada. J Clin Microbiol 2000; 3: 949-53.

21.- Testore G, Dori L, Buonomini A, Schito G, Soro O, Fortina G, et al. In vitro fluconazole susceptibility of 1565 clinical isolates of Candida species evaluated by the disk diffusion method performed using NCCLS M44-A guidelines. Diagn Microbiol Infect Dis 2004; 50: 187-92.

22.- Pfaller M, Jones R, Doern G, Sader H, Hollis R, Messer S. International surveillance of bloodstream infections due to Candida species: frequency of occurrence and antifungal susceptibilities of isolates collected in 1997 in the United States, Canada, and South America for the SENTRY program. J Clin Microbiol 1998; 36: 1886-9.

23.- Pfaller M, Jones R, Doern G, Sader H, Messer S, Houston A. Bloodstream infections due to Candida species: SENTRY antimicrobial surveillance program in North America and Latin America 1997-1998. Antimicrob Agents Chemother 2000; 44: 747-51.

24.- Pfaller M, Diekema D, Gibbs D, Newell V, Meis J, Gould I, et al. Results from the ARTEMIS DISK Global Antifungal Surveillance Study, 1997 to 2005: an 8.5year analysis of susceptibilities of Candida species and other yeast species to fluconazole and voriconazole determined by CLSI standardized disk diffusion testing. J Clin Microbiol 2007; 45: 1735-45.

25.- Rivas J, Cortés J, Cuervo S, Vanegas E, Bermúdez D. Resistencia al fluconazol en aislamientos clínicos de Candida en un centro de referencia de pacientes con cáncer. Libro de Resúmenes V Congreso 
Panamericano de Control de Infecciones y Epidemiología Hospitalaria y I Congreso Peruano de Control de Infecciones Hospitalarias. Rev Peruana Enf Infec Trop 2004. 7-10 oct. Lima-Perú.

26.- De Bedout C, Ayabaca J, Vega R, Méndez M, Santiago A, Pabón M. Evaluación de la susceptibilidad de especies de Candida al fluconazol por el método de difusión de disco. Biomédica 2003; 23: 31-7.

27.- Villar L, Díaz F, Céspedes J, Torres A, De Bedout C. Patrones de sensibilidad y resistencia de asilamiento de Candida en pacientes críticos de la ciudad de Bucaramanga. Infectio 2002; 6 : E-12.

28.- Gutiérrez C, De Bedout C, Tobón A, Cano L, Arango M, Tabares A, et al. Sensibilidad al fluconazol y voriconazol de aislamientos de Candida spp, obtenidos de mucosa oral de pacientes con SIDA. Infectio 2007; 11: 183-9.

29.- Tortorano A, Rigoni A, Biraghi E,
Prigitano A, Viviani M, FIMUA-ECMM candidaemia study group. The European Confederation of Medical Mycology (ECMM) survey of candidaemia in Italy: antifungal susceptibility patterns of 261 non-albicans Candida isolates from blood. J Antimicrob Chemother 2003; 52: 679-82.

30.- Silva V, Díaz M, Febrè N y red de diagnóstico en micología médica. Vigilancia de la resistencia de levaduras a antifúngicos. Rev Chil Infec 2002; 19: S149-56.

31.- Colombo AL, Barchiesi F, McGough DA, Rinaldi MG. Comparison of E-test and National Committee for Clinical Laboratory Standards broth macrodilution method for azole antifungal susceptibility testing. J Clin Microbiol 1995; 33: 535-40.

32.- Gil J, Rubio C, Benito R. Criterios de sensibilidad a los azoles. Rev Esp Quimioterap 2004; 17: 83-90

33. - Capoor M, Nair D, Deb M, Verma P, Srisvastava L, Aggarwal P. Emergence of
non-Candida species and antifungal resistance in a tertiary care hospital. Jpn J Infect Dis 2005; 58: 344-8.

34.- Finquelievich J, Iovannitti C, Mujica M, Rellos S, De Elias Costa M, Jewtuchowicz V, et al. Susceptibility of Candida species to fluconazole assayed by a disk diffusion method with automated reading versus a microdilution method. Rev Argent Microbiol 2003; 35: 214-8.

35.- Pfaller M, Liang Yu W. Antifungal susceptibility testing. Infect Dis Clin North Am 2001; 15: 1227-61.

36.- Pfaller M, Diekema D, Rinaldi M, Barnes R, $\mathrm{Hu}$ B, Veselov A, et al. and the Global Antifungical Surveillance Group. Results from ARTEMIS DISK Global Antifungal Surveillance study: a 6.5-year analysis of susceptibilities of Candida and other yeast species to fluconazol and voriconazol by standardized disk diffusion testing. J Clin Micorbiol 2005; 43: 5848-59. 